\title{
rasiRNAs, DNA Damage, and Embryonic Axis Specification
}

\author{
W.E. Theurkauf, ${ }^{*}$ C. Klattenhoff, ${ }^{*}$ D.P. Bratu, $*$ N. McGinnis-Schultz, ${ }^{*}$ \\ B.S. KOPPETSCH, ${ }^{*}$ AND H.A. COOK ${ }^{\dagger}$ \\ *Program in Molecular Medicine and Program in Cell Dynamics, University of Massachusetts Medical School, \\ Worcester, Massachusetts 01605; 'Department of Biological Sciences, Wagner College, Staten Island, New York 10301
}

\begin{abstract}
Drosophila repeat-associated small interfering RNAs (rasiRNAs) have been implicated in retrotransposon and stellate locus silencing. However, mutations in the rasiRNA pathway genes armitage, spindle- $E$, and aubergine disrupt embryonic axis specification, triggering defects in microtubule organization and localization of $o s k$ and grk mRNAs during oogenesis. We show that mutations in $m e i-41$ and $m n k$, which encode ATR and Chk2 kinases that function in DNA damage signal transduction, dramatically suppress the cytoskeletal and RNA localization defects associated with rasiRNA mutations. In contrast, stellate and retrotransposon silencing are not restored in mei-41 and mnk double mutants. We also find that armitage, aubergine, and spindle- $E$ mutations lead to germ-line-specific accumulation of $\gamma-\mathrm{H} 2 \mathrm{Av}$ foci, which form at DNA double-strand breaks, and that mutations in armi lead to Chk2-dependent phosphorylation of Vasa, an RNA helicase required for axis specification. The Drosophila rasiRNA pathway thus appears to suppress DNA damage in the germ line, and mutations in this pathway block axis specification by activating an ATR/Chk2-dependent DNA damage response that disrupts microtubule polarization and RNA localization.
\end{abstract}

RNA interference (RNAi) and related processes utilize short RNAs to direct protein complexes to chromatin or RNA, triggering heterochromatin formation, transcriptional silencing, translational repression, or RNA destruction (Hannon 2002; Hutvagner and Zamore 2002; Wassenegger 2005). Mutations in genes involved in small RNA function lead to embryonic lethality in mice (Bernstein et al. 2003), disrupt embryo morphogenesis in zebra fish (Giraldez et al. 2005), and lead to defects in developmental timing in worms (Grishok et al. 2001). Mutations in RNAi-related processes also interfere with stem cell division, stem cell maintenance, and viral immunity in flies (Forstemann et al. 2005; Hatfield et al. 2005; Galiana-Arnoux et al. 2006; Wang et al. 2006), and block chromosome segregation in cultured chicken cells (Fukagawa et al. 2004) and yeast (Provost et al. 2002; Volpe et al. 2003). However, the full scope of biological functions controlled by small RNAs is only beginning to emerge, and new classes of these RNAs continue to be discovered (Aravin et al. 2006; Girard et al. 2006; Vagin et al. 2006). Moreover, the targets for most small RNAs have not been identified, and the molecular basis for the phenotypes associated with small RNA production and function are not well understood.

Mutations in the Drosophila armitage (armi), spindleE (spn-E), and aubergine (aub) genes disrupt siRNAguided RNA cleavage and assembly of the RNA-induced silencing complex (RISC) in ovary extracts and production of 24- to 30-nucleotide repeat-associated siRNAs (rasiRNAs), which are linked to retrotransposon and Stellate locus silencing (Aravin et al. 2004; Tomari et al. 2004b; Vagin et al. 2006). Strong loss-of-function mutations in these genes disrupt embryonic axis specification, triggering defects in microtubule organization and microtubule-dependent localization of mRNA and protein determinants in the developing oocyte (Cook et al. 2004).
In contrast, mutations in argonaute-2 (ago-2) and dicer-2 $(d c r-2)$ that disrupt the siRNA pathway, but do not block rasiRNA production, are viable and fertile (Lee et al. 2004; Okamura et al. 2004; Tomari et al. 2004a; Deshpande et al. 2005; Vagin et al. 2006). The rasiRNA pathway thus appears to have an essential function in embryonic axis specification; however, the critical developmental targets for this pathway have not been defined.

The axes of the Drosophila embryo are specified through asymmetric localization of RNA and protein determinants within the oocyte, and this complex process presents a number of potential targets for rasiRNA control. Oogenesis begins in the germarium with a stem cell division that produces a cystoblast, which divides four times with incomplete cytokinesis to generate a cyst of 16 germ-line cells interconnected by ring canal junctions (Spradling 1993). The cysts reorganize and are surrounded by somatic follicle cells as they pass through the germarium. The pro-oocyte is at the posterior of cysts in region 3 of the germarium. A single microtubule organizing center (MTOC) is present at the anterior pole of the pro-oocyte, and microtubules extend from this MTOC into the 15 pro-nurse cells (Theurkauf et al. 1992). As the germ line/somatic cell complexes bud from region 3 to form stage 2 egg chambers, the MTOC re-forms at the posterior cortex of the oocyte (Theurkauf et al. 1992; Riechmann and Ephrussi 2001). The posterior MTOC directs gurken (grk) mRNA and protein to the posterior pole. Grk protein is a TGF $\alpha$ homolog that signals to the somatic follicle cells, which leads to posterior differentiation (Gonzalez-Reyes et al. 1995; Roth et al. 1995). During mid-oogenesis, the posterior follicle cells signal back to the oocyte, inducing a reorganization of the oocyte microtubule cytoskeleton that leads to oskar mRNA localization to the posterior pole and dorsal-anterior localization of Grk, which signals to the dorsal folli- 
cle cells to trigger dorsal differentiation (NeumanSilberberg and Schupbach 1993; Gonzalez-Reyes et al. 1995; Roth et al. 1995). Microtubule polarization and Grk localization during early oogenesis thus initiate embryonic axis specification.

Mutations in the rasiRNA pathway lead to premature expression of Osk protein during early oogenesis (Cook et al. 2004), suggesting that the axis specification defects associated with these mutations may result from defects in expression of axis specification genes. However, here we show that the cytoskeletal polarization and morphogen localization defects associated with armi and $a u b$ are dramatically suppressed by null mutations in mei-41 and $m n k$, which encode ATR and Chk2 kinases that function in DNA double-strand-break (DSB) signaling. We also show that rasiRNA pathway mutations lead to germ-line-specific accumulation of $\gamma-\mathrm{H} 2 \mathrm{Av}$ foci characteristic of DNA DSBs. Significantly, the ATR/Chk2 mutations do not suppress the defects in retrotransposon and Stellate silencing. We therefore conclude that rasiRNA-based gene silencing is not required for axis specification, and that the critical developmental function for the Drosophila rasiRNA pathway is to maintain germ-line genome integrity. Mutations in the rasiRNA pathway thus lead to DNA damage, which activates an ATR and Chk2 kinase pathway that blocks axis specification by disrupting microtubule polarization and asymmetric RNA localization.

\section{ATR AND CHK2 MUTATIONS SUPPRESS ARMI AND $A U B$ AXIS SPECIFICATION DEFECTS}

The $a r m i, s p n-E$, and $a u b$ genes are required for production of rasiRNAs and lead to Stellate overexpression during spermatogenesis and premature Oskar protein expression during oogenesis (Aravin et al. 2001; Cook et al. 2004; Vagin et al. 2006). Mutations in these genes lead to female sterility and disrupt embryonic axis specification, suggesting that rasiRNAs control expression of genes involved in patterning the oocyte (Cook et al. 2004). However, mutations in the meiotic DSB repair pathway also lead to axis specification defects through activation of a posttranslational DNA damage response (Ghabrial et al. 1998). In these mutants, persistent meiotic DSBs activate a damage signaling pathway that includes the ATR and Chk2 kinases, which induce the observed axis specification defects (Ghabrial and Schupbach 1999; Bartek et al. 2001; Abdu et al. 2002). These findings raised the alternative possibility that rasiRNA pathway mutations disrupt axis specification by activating the ATR and Chk2 pathway.

To genetically test the role of DNA damage signaling in the rasiRNA pathway mutant phenotype, we analyzed double mutant combinations with mei-41 or mnk, which encode the Drosophila ATR and Chk2 homologs. We were unable to recover $m n k$; spn-E double mutants, and it is unclear whether this reflects a significant negative genetic interaction between these genes or is the result of background mutations on the mnk or spn-E chromosome. Our analyses thus focused on armi and $a u b$, which we were able to combine with mei-41 and mnk. If armi and aub mutations block axis specification through ATR/Chk2 activation, the patterning defects associated with these mutations will be suppressed in the double mutants. Initial suppression analysis focused on the dorsal appendages, which are easily scored eggshell structures that are induced through Grk signaling from the oocyte to the somatic follicle cells during mid-oogenesis (Schupbach 1987). Appendages do not form in the absence of Grk, a single appendage forms with low Grk levels, and two appendages form when signaling is normal (GonzalezReyes et al. 1995; Roth et al. 1995). As shown in Table 1, $m e i-41$ and $m n k$ dramatically suppress the appendage defects associated with armi and $a u b$. Two appendages are present on $100 \%$ of the embryos derived from wild-type and mei-41 females, and on $94 \%$ of the embryos derived from mnk single mutants (Table 1). In contrast, only $3.5 \%$ of the embryos derived from armi ${ }^{72.1} /$ armi $^{1}$ mutant females have two dorsal appendages. Strikingly, 92\% of the embryos derived from $m n k$; armi $^{72.1} /$ armi $^{1}$ double mutants show wild-type appendage morphology.

Table 1. mnk and mei-41 Mutations Suppress Dorsal-Ventral Patterning Defects in rasiRNA Mutants

\begin{tabular}{|c|c|c|c|c|c|}
\hline \multirow[b]{2}{*}{ Maternal genotype } & \multicolumn{3}{|c|}{ Dorsal appendage (\%) phenotype } & \multirow{2}{*}{$\begin{array}{c}\text { Hatch rate } \\
(\%)\end{array}$} & \multirow[b]{2}{*}{$\mathrm{N}$} \\
\hline & 2 (wild type) & 1 (fused) & 0 (absent) & & \\
\hline$m n k P 6 / m n k P 6$ & 94.1 & 2.3 & 3.6 & 73.9 & 827 \\
\hline mei41D3 / mei41D3 & 100 & 0 & 0 & 0 & 920 \\
\hline meiW681 / meiW68K05603 & 94.3 & 4 & 1.7 & 67.2 & 1281 \\
\hline $\operatorname{armi} 72.1$ / armil & 3.5 & 67.6 & 28.9 & 0 & 765 \\
\hline mnkP6 / mnkP6 ; armi72.1 / armil & 91.9 & 2.5 & 5.6 & 0 & 1062 \\
\hline mei41D3 / mei41D3 ; armi72.1 / armil & 56 & 38.4 & 5.6 & 0 & 575 \\
\hline meiW681 / meiW68K05603 ; armi72.1 / armil & 3.6 & 37.9 & 58.5 & 0 & 280 \\
\hline aubHN2 / aubQC42 & 47.7 & 40.3 & 12 & 0 & 1212 \\
\hline mnkP6, aubHN2 / mnkP6, aubQC42 & 97.6 & 2 & 0.4 & 0 & 296 \\
\hline mei41D3 / mei41D3; aubHN2/ aubQC42 & 85.2 & 8.6 & 6.2 & 0 & 859 \\
\hline spn-E1 / spn-E1 & 16.6 & 55.4 & 27.9 & 0 & 123 \\
\hline mei41D3 / mei41D3 ; spn-E1 / spn-E1 & 23.9 & 56.2 & 19.9 & 0 & 233 \\
\hline spn-D2 / spn-D2 & 34.8 & 54 & 11.2 & 17.3 & 1245 \\
\hline mnkP6 / mnkP6 ; spn-D2 / spn-D2 & 98.8 & 0.5 & 0.7 & 45.9 & 812 \\
\hline
\end{tabular}

Two dorsal appendages are normally present at the dorsal side of a wild-type Drosophila egg. The mutant phenotypes are classified as weakly ventralized, which results in fusion of the dorsal appendages, and strongly ventralized, resulting in absence of dorsal appendages. 
Similarly, two appendages are present on $48 \%$ of embryos derived from aub single mutants, and $98 \%$ of the embryos from $m n k$, aub double mutants have two appendages.

Mutations in mei-41 also suppressed the eggshell patterning defects associated with armi and $a u b$ mutations, although suppression by mei-41 was consistently less dramatic than suppression by mnk. Of the embryos from $m e i$ 41; armi $^{72.1} /$ armi $^{1}$ double mutants, 56\% show normal appendages. The mei-41 mutation was also less effective than $m n k$ in suppressing appendage defects associated with homozygous armi $^{1}$ (data not shown) and aub (Table $1)$, indicating that this difference is not gene- or allelespecific. Chk2 can be activated by both ATR and ATM kinases (Bartek et al. 2001; Hirao et al. 2002; Bartek and Lukas 2003), and the lower level of suppression by mei$41 / \mathrm{ATR}$ relative to $m n k / \mathrm{Chk} 2$ may therefore reflect redundant Chk2 activation by the Drosophila ATM homolog. However, null alleles of the Drosophila atm gene are lethal (Oikemus et al. 2004), making direct tests of this hypothesis difficult. Nonetheless, these initial observations suggested that the axis specification defects associated with rasiRNA pathway mutations result from activation of an ATR/Chk2 kinase DNA damage signal.

The axis specification defects associated with repair mutations are suppressed by mutations in mei-W68, which encodes the Drosophila homolog of the Spo11 nuclease that catalyzes meiotic DSB formation. In contrast, meiW68 has no effect on the dorsal appendage defects associated with armi (Table 1). Meiotic breaks thus do not appear to be the source of damage in armi mutations.

\section{LOCALIZATION OF AXIS SPECIFICATION DETERMINANTS}

During early oogenesis, the TGF $\alpha$ homolog Grk localizes to the posterior of the oocyte and signals to the overlying follicle cells, inducing posterior differentiation. During mid-oogenesis, Grk signals from the oocyte to the dorsal follicle cells to generate the dorsal-ventral axis (Gonzalez-Reyes et al. 1995; Roth et al. 1995). Mutations in armi and $a u b$ disrupt Grk protein localization at both stages, leading to posterior and dorsal-ventral axis specification defects (Cook et al. 2004). To determine whether the DNA damage signaling mutations suppress these defects in Grk localization, we analyzed the distribution of this protein by indirect immunofluorescence and laser scanning confocal microscopy (Fig. 1). For these studies, Grk protein levels within cross sections of stage 6 oocytes were measured, and an average fluorescence intensity profile for each genotype was generated (Fig. 1B, inset). In wild-type stage- 6 oocytes, Grk protein accumulates near the posterior cortex (Fig. 1A, panel a). In armi and $a u b$ single mutants, in contrast, Grk protein is more uniformly distributed in the oocyte and nurse cells (Fig. 1A, $\mathrm{c}$ and e). However, Grk shows almost wild-type accumulation near the posterior cortex of $m n k$; armi and $m n k, a u b$ double mutant oocytes (Fig. 1A, d and f). The defects in dorsal-anterior localization of Grk during mid-oogenesis (Fig. 1A, panels $\mathrm{c}^{\prime}$ and $\mathrm{e}^{\prime}$ ) are also restored in the $m n k$ double mutants (Fig. 1A, $\mathrm{d}^{\prime}$ and $\mathrm{f}^{\prime}$ ). Weaker suppression is observed with mei-41, consistent with our analysis of the dorsal appendages (not shown).

To determine whether mnk and mei-41 suppress the armi and $a u b$ induced defects in posterior morphogen localization (Cook et al. 2004), we analyzed the distribution of the pole plasm proteins Vasa (Vas) and Oskar (Osk) during mid-oogenesis. Osk localizes to the posterior in only $10 \%$ of stage- 9 and stage- 10 oocytes from armi females ( 2 of 23), with no detectable localization in the remaining egg chambers (Fig. 2c). In contrast, Osk shows wild-type posterior accumulation in over $80 \%$ of stage- 9 and stage-10 mnk; armi double mutants (27 of 33; Fig. 2d). Vas localization to the posterior pole is similarly restored in the double mutants (not shown). mei-41 leads to a less dramatic suppression of the posterior patterning defects (not shown). Osk and Vas localization are also disrupted in $a u b$ mutants (Fig. 2e and data not shown), and localization is restored in double mutants with $m n k$ and mei-41 (Fig. 2f). The defects in posterior and dorsal-ventral morphogen localization associated with both armi and aub thus require ATR and Chk2, which function in DNA damage signal transduction.

\section{ATR AND CHK2 MUTATIONS RESTORE MICROTUBULE POLARIZATION}

Specification of the posterior pole is initiated during early oogenesis, when the microtubule cytoskeleton reorganizes to form a polarized scaffold in the oocyte nurse cell complex. When these complexes are in the germarium, a prominent MTOC forms at the anterior pole of the oocyte, and this MTOC appears to be required for oocyte differentiation (Theurkauf et al. 1993). After cysts bud from the germarium, a posterior MTOC is established (Fig. 3a'). This asymmetric microtubule array directs Grk to the posterior pole of the oocyte, which signals to the overlying somatic follicle cells to induce posterior differentiation (Gonzalez-Reyes et al. 1995; Roth et al. 1995). Mutations in armi disrupt the posterior MTOC (Fig. 3b') (Cook et al. 2004). In contrast, egg chambers double mutant for armi and mnk show near-wild-type microtubule organization at both stages (Fig. $3 c^{\prime}$ ). This correlates with restoration of normal posterior localization of Grk protein (Fig. 1A, d). Egg chambers double mutant for armi and mei-41 show a phenotype intermediate between the armi mutants and wild-type controls, consistent with partial suppression of posterior patterning defects later in oogenesis. Microtubule organization defects in aub are also strongly suppressed by mnk and more weakly suppressed by mei-41 (data not shown). Mutations in armi and $a u b$ thus trigger Chk2-dependent defects in microtubule organization. Interestingly, DNA-damage-dependent Chk2 activation disrupts mitotic MTOCs in syncytial embryos (Takada et al. 2003). Chk2, which has an established function in control of the cell cycle machinery, thus appears to control microtubule organization during oogenesis and embryogenesis.

The axis specification defects in meiotic DSB repair mutations are also suppressed by $m n k$, suggesting that these mutations trigger Chk2-dependent defects in micro- 
A.

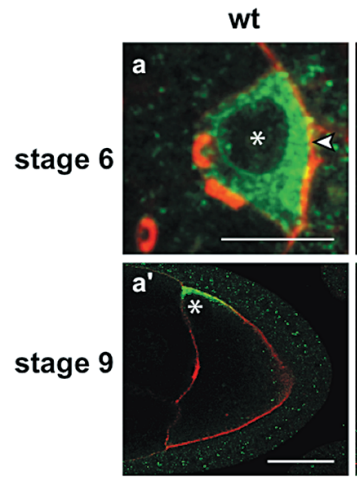

$m n k$
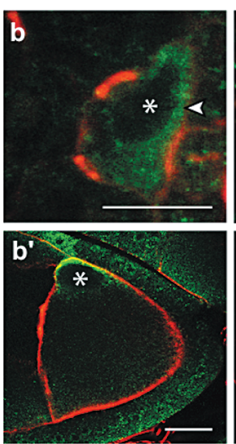

armi
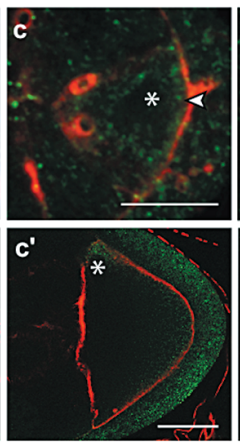

mnk;armi
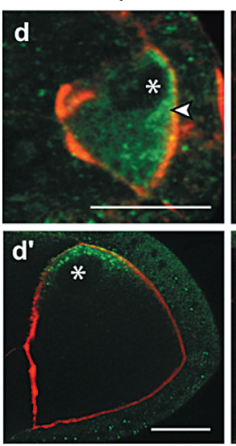

aub
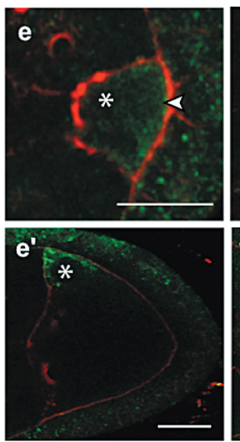

$m n k, a u b$
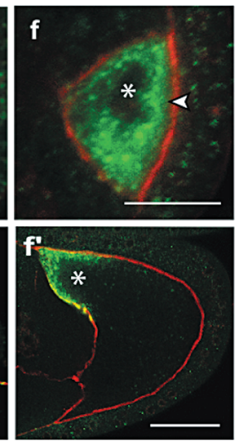

anterior $\longrightarrow$ posterior

B.

Gurken distribution at stage 6

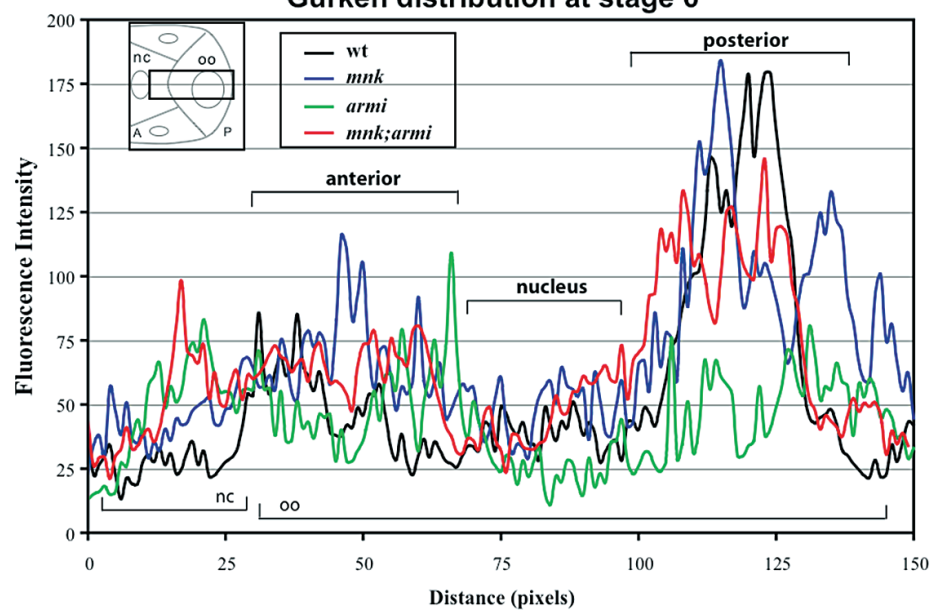

Figure 1. mnk restores Gurken protein localization in rasiRNA mutants. $(A)(a)$ In wild-type stage-6 egg chambers, Gurken (Grk) protein (green) is tightly localized at the posterior cortex (arrowhead) near the oocyte nucleus (asterisk). ( $\left.a^{\prime}\right)$ By stage 9 , Grk is localized at the dorsal anterior cortex near the oocyte nucleus (asterisk). Actin filaments $(r e d)$ mark the cell boundaries. $\left(b, b^{\prime}\right)$ In $m n k^{p 6}$ oocytes, Grk localization is the same as in wild type. $\left(c, c^{\prime}\right)$ In $a r m i^{72.1} / a r m i^{I}$ egg chambers, this localization pattern is lost, with Grk dispersed throughout the oocyte. $\left(d, d^{\prime}\right) m n k^{p 6}$ suppresses the $a r m i^{72.1} / a r m i^{l}$ phenotype and rescues Grk localization during early and late oogenesis. (e) In $a u b^{Q C 42} / a u b^{H N 2}$ stage-6 egg chambers, Grk localization is similar to that of $a r m i^{72.1} / a r m i i^{1}$ oocytes. ( $\left.e^{\prime}\right)$ At stage 9, Grk is localized correctly in $a u b^{Q C 42} / a u b^{H N 2}$, but not at wild-type levels. $\left(f, f^{\prime}\right)$ In $m n k^{p 6}$, aub $b^{Q C 42} / m n k^{p 6}$, aub $b^{H N 2}$ egg chambers, Grk localization level is restored. Images were acquired under identical conditions for either stage. Projections of three serial $0.6-\mu \mathrm{m}$ optical sections are shown. Scale bars, $10 \mu \mathrm{m}$ and $25 \mu \mathrm{m}$ for stage- 6 and -9 egg chambers, respectively. $(B)$ Quantification of Gurken localization in stage- 6 oocytes. Distribution of fluorescence was measured within a region comprising some nurse cell cytoplasm and a cross section of the oocyte (inset). Single Z-section images were acquired focusing on both the nucleus and the posterior cortex. The measurements for each genotype were performed post-acquisition using Image J software and averaged over 4-5 oocytes per genotype using Microsoft Excel.

tubule polarization during early oogenesis. We therefore analyzed microtubule organization in ovaries mutant for $s p n-D$, which encodes a rad51C homolog required for DSB repair (Abdu et al. 2003). Mutations in $s p n-D$, like mutations in armi and $a u b$, disrupt formation of both a prominent MTOC at the anterior of stage 1 egg chambers, and of the posterior MTOC during stages 2 through 6 (Fig. $3 \mathrm{~d}$ and $\left.\mathrm{d}^{\prime}\right)$. Significantly, these defects are suppressed in $m n k$; spn-D double mutants (Fig. $3 \mathrm{e}$ and $\mathrm{e}^{\prime}$ ). Therefore, both DNA repair and rasiRNA mutations lead to defects in microtubule polarization that are mediated by Chk 2 .

Repair mutations induce Chk2-dependent phosphorylation of Vas, a conserved RNA helicase required for posterior and dorsal-ventral patterning (Styhler et al. 1998; Ghabrial and Schupbach 1999). To determine whether rasiRNA mutations also trigger Chk2-dependent Vas phosphorylation, we probed western blots of armi and mnk; armi double mutants for this conserved helicase. As shown in Figure 4, a single Vas species is observed on blots of ovary extracts from wild type and homozygous $\mathrm{armi}^{1}$ mutants. In contrast, a single lower electrophoretic mobility species is observed in ovaries homozygous for a stronger loss-of-function allele of armi $\left(a_{r m i} i^{72.1}\right)$. Both species are observed with an inter-allelic combination $\left(a r m i^{72.1} / a r m i^{1}\right)$. We were unable to recover $m n k$; $a r m i^{72.1}$ double mutants. However, only the fastermigrating species is present in $m n k$; armi $^{72.1} /$ armi $^{1}$ double mutant extracts. Following phosphatase treatment, the lower-mobility species present in armi mutant extracts disappears and the faster-migrating species increases in intensity (data not shown), indicating that the lowermobility band is a phosphorylated form of Vas. Mutations in armi, like meiotic DSB repair mutations, thus trigger Chk2-dependent phosphorylation of Vas. 

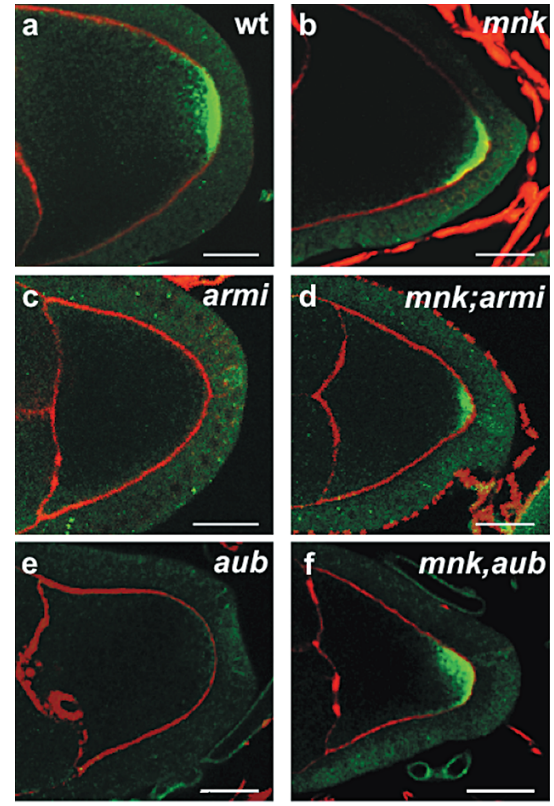

anterior $\longrightarrow$ posterior

Figure 2. $m n k$ restores Oskar protein localization during late oogenesis in rasiRNA mutants. Egg chambers were fixed and labeled against Oskar protein (green) and Actin (red). (a) In wild-type stage 9-10 oocytes, Oskar (Osk) localizes tightly to the posterior cortex. (b) $m n k^{p 6}$ mutants have no defects in Osk localization. (c,e) In $a r m i^{72.1} / a r m i^{1}$ and $a u b^{Q C 42} / a u b^{H N 2}$ stage-9 oocytes, Osk localization is disrupted. $(d, f) m n k^{p 6}$ rescues the Osk localization in $a r m i^{72.1} / a r m i^{I}$ and $a u b^{Q C 42} / a u b^{H N 2}$ stage-9 oocytes. Egg chambers are oriented with posterior to the right. Images were acquired under identical conditions. Single optical sections are shown. Scale bar, $20 \mu \mathrm{m}$.

\section{ARMI AND AUB MUTATIONS LEAD TO GERM- LINE $\gamma$-H2AV ACCUMULATION}

The above observations indicate that the axis specification defects in armi and $a u b$ mutants require ATR and Chk2 kinases that can be activated by DNA DSBs. However, the armi mutation is not suppressed by mei$W 68$, suggesting that meiotic breaks are not the source of DNA damage. To determine whether the armi and $a u b$ mutations lead to DSB accumulation, we labeled mutant ovaries for the phosphorylated form of the Drosophila histone $\mathrm{H} 2 \mathrm{AX}$ variant ( $\gamma-\mathrm{H} 2 \mathrm{Av})$, which accumulates on chromosomes near break sites (Modesti and Kanaar 2001; Redon et al. 2002). Following chromosome breakage, Drosophila $\mathrm{H} 2 \mathrm{Av}$, like $\mathrm{H} 2 \mathrm{AX}$, is phosphorylated at a conserved SQ motif within an extended carboxy-terminal tail (Rogakou et al. 1998; Madigan et al. 2002). For these studies, we used an anti-phosphoprotein antibody specific for $\gamma$-H2Av (Gong et al. 2005). As shown in Figure 5a, $\gamma$ $\mathrm{H} 2 \mathrm{Av}$ foci are normally restricted to region 2 of the germarium, where meiotic DSBs are formed (Jang et al. 2003). Consistent with earlier observations, this labeling is significantly reduced in mei-W68 mutants, which do not initiate meiotic breaks (Fig. 5g). In armi and $a u b$ mutants, prominent $\gamma-\mathrm{H} 2 \mathrm{Av}$ foci are present in germ-line cells of the germarium. However, these foci persist and increase in intensity as cysts mature and bud to form stage- 2 egg chambers (Fig. 5c and e). $\gamma$-H2Av foci also persist in spn$E$ mutants, which are defective in rasiRNA function and trigger axis specification defects (data not shown).

The pattern of germ-line-specific $\gamma-\mathrm{H} 2 \mathrm{Av}$ accumulation in $a r m i, a u b$, and $s p n-E$ is similar to the pattern observed in the DNA repair mutant $s p n-D$, although the wt
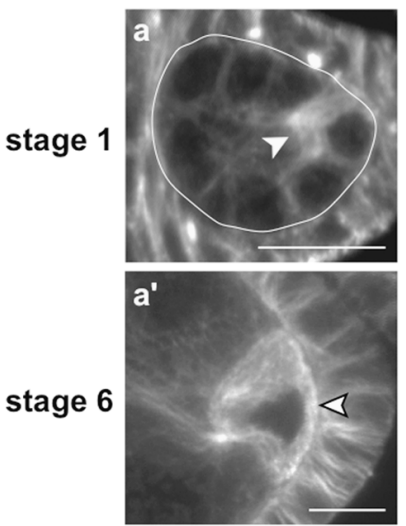

armi
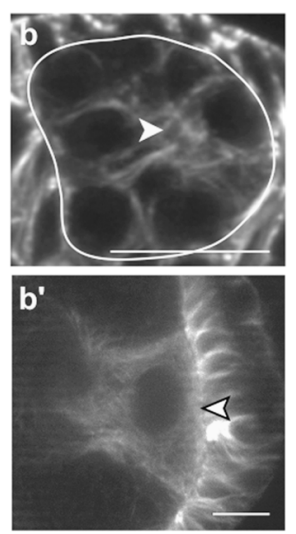

mnk; armi
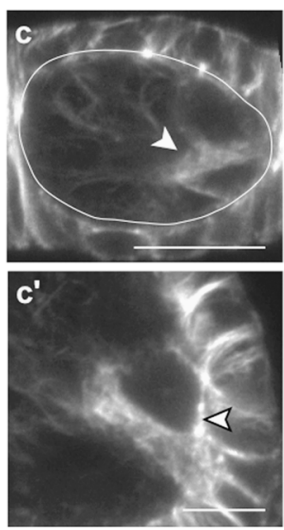

spn-D
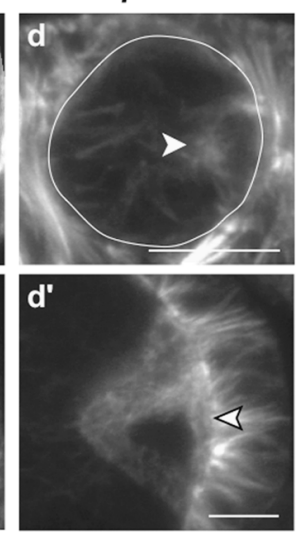

$m n k ; s p n-D$
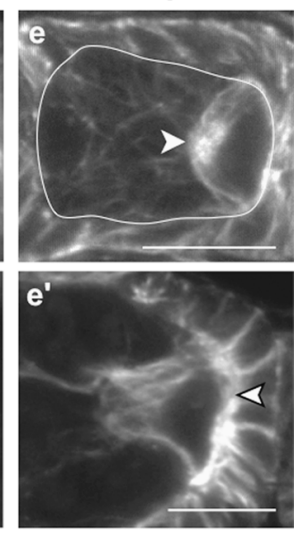

anterior $\longrightarrow$ posterior

Figure 3. mnk suppresses the microtubule organization defects caused by mutations in the rasiRNA and DNA repair pathways. $\left(a, a^{\prime}\right)$ Wild type, $\left(b, b^{\prime}\right) a r m i^{72.1} / a r m i i^{1},\left(c, c^{\prime}\right) m n k^{p 6} ; a r m i^{72.1} / a r m i^{I},\left(d, d^{\prime}\right) s p n-D^{2}$, and $\left(e, e^{\prime}\right) m n k^{p 6} ; s p n-D^{2}$ egg chambers were fixed and labeled with FITC-conjugated anti- $\alpha$-tubulin antibody. ( $a$ ) A bright MTOC is localized to the anterior pole of the oocyte in wild-type stage-1 egg chambers (arrowhead). $\left(a^{\prime}\right)$ By stage 6 , the MTOC is localized along the posterior cortex $($ arrowhead). $(b, d)$ In armi ${ }^{72.1} / a r m i ~ a n d ~ s p n-D^{2}$ mutant egg chambers, the stage-1 anterior MTOC and later posterior MTOC $\left(b^{\prime}, d^{\prime}\right)$ are disrupted (arrowheads). $(c, e)$ In $m n k^{p 6} ; a r m i^{72.1} / a r m i^{1}$, and $m n k^{p 6} ; s p n-D^{2}$ egg chambers, the MTOC is observed at the anterior of the oocyte during stage 1 (arrowheads) and $\left(c^{\prime}, e^{\prime}\right)$ at the posterior of the oocyte in stage 6 (arrowheads). Stage-1 oocytes are outlined. Images were acquired under identical conditions. Projections of four serial $0.6-\mu \mathrm{m}$ optical sections are shown. Posterior is oriented to the right. Scale bar, $10 \mu \mathrm{m}$. 


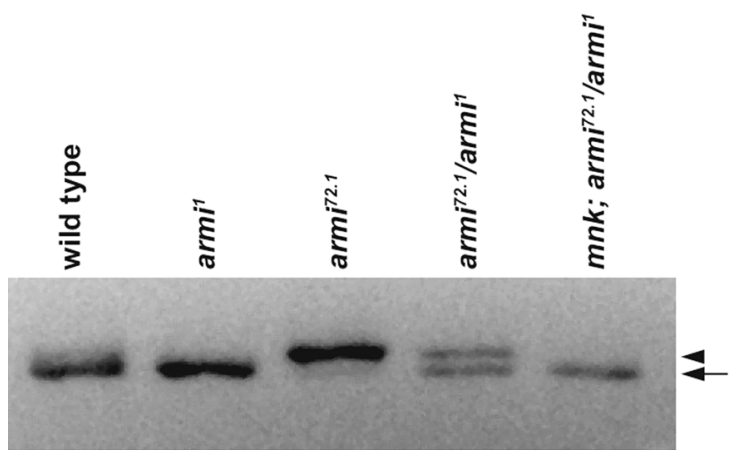

Figure 4. Severe armi mutations trigger $m n k$-dependent Vas phosphorylation. Western blot analysis of Vas protein in wild type, armi ${ }^{1}, \operatorname{armi}^{72.1}, \operatorname{armi}^{72.1} / \mathrm{armi}^{1}$, and $m n k^{p 6} ; \operatorname{armi}^{72.1} / \mathrm{armi}^{1}$ ovary extracts. Vas from homozygous $a r m i^{72.1}$ ovaries has a reduced electrophoretic mobility relative to Vas from wild-type ovaries. Low-mobility and wild-type-mobility forms of Vas are present in $\mathrm{armi}^{72.1} / \mathrm{armi}^{1}$ ovary extracts. Only the faster-migrating form is present in $m n k^{p 6}$; armi $i^{72.1} /$ armi $^{1}$ extracts (arrow). Following phosphatase treatment, the lower-mobility species in armi mutants is lost and the faster-migrating form increases in intensity, indicating that the lower-mobility form is phosphorylated (not shown)

foci appear to arise at somewhat earlier stages in the rasiRNA mutants (Fig. 5c, e, i, j). DSB formation in spn$D$ mutants is suppressed by $m e i-W 68$ (Abdu et al. 2002). Consistent with the observation that mei-W68 does not suppress the dorsal-ventral axis specification defects linked to armi (Table 1), $\gamma-\mathrm{H} 2 \mathrm{Av}$ foci persist in meiW68; armi double mutants (Fig. 5h). Mutations in armi, $a u b$, and $s p n-E$ thus appear to trigger DNA DSBs in the germ line. Our analysis of mei-W68; armi double mutants strongly suggests that this is independent of meiotic DSB formation.

\section{RASIRNA FUNCTION}

The observations presented above strongly suggested that the axial patterning defects associated with armi and $a u b$ are a consequence of DNA damage signaling, and that rasiRNA-based gene silencing is not directly involved in embryonic patterning. Alternatively, the $m n k$ and mei-41 mutations could suppress the defects in rasiRNA function associated with armi and $a u b$. If this were the case, rasiRNA-dependent silencing could be essential to axis specification. We therefore analyzed rasiRNA-dependent silencing of both the Stellate (Ste) gene during spermatogenesis and the HeT-A retrotransposon during oogenesis in single and double mutants. The Ste gene is repressed during spermatogenesis, apparently through mRNA turnover guided by rasiRNAs derived from the Suppressor of Stellate locus (Aravin et al. 2001; Gvozdev et al. 2003). Mutations in armi and aub lead to accumulation of full-length Ste mRNA and Stellate protein overexpression, triggering assembly of Ste crystals in mutant testes (Fig. 6A, b and c) (Stapleton et al. 2001; Aravin et al. 2004; Tomari et al. 2004b; Forstemann et al. 2005). We found that Ste crystals are present in both mnk; armi and mnk, aub double mutant testes (Fig. 6A, e and f). Ste overexpression is also linked to male sterility, and mnk; armi males are sterile (data not shown). $H e T-A$ is a retrotransposon that contributes to
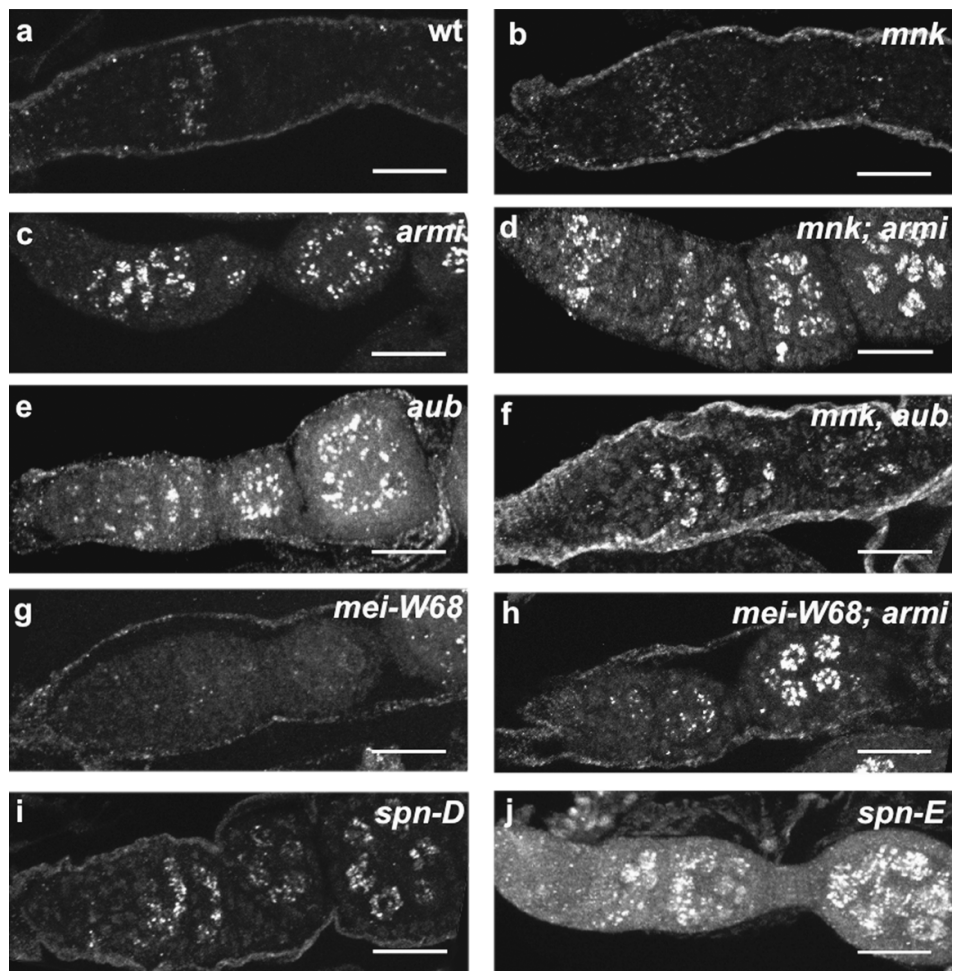

Figure 5. $\gamma-\mathrm{H} 2 \mathrm{Av}$ foci accumulate in armi and $a u b$ mutant ovaries. The phosphorylated form of histone $\mathrm{H} 2 \mathrm{Av}(\gamma-\mathrm{H} 2 \mathrm{Av})$ accumulates near DSB sites. $(a, b)$ In wild type and $m n k^{p 6}$ mutants, $\gamma$ $\mathrm{H} 2 \mathrm{Av}$ foci are restricted to region 2 of the germarium, when meiotic DSBs are present. In $(c)$ $\operatorname{armi}^{72.1} / \operatorname{armi}^{1},(d) m_{n} k^{p 6} ; \operatorname{armi}^{12.1} / \operatorname{armi}^{1},(e)$ $a u b^{Q C 42} / a u b^{H N 2},(f) m n k^{p 6}, a u b^{Q C 42} / m n k^{p 6}$, $a u b^{H N 2}$, and (j) spn-E mutant ovaries, $\gamma-\mathrm{H} 2 \mathrm{Av}$ foci accumulate in germ-line cells within the germarium, and persist and increase in intensity as cysts bud from the germarium to form egg chambers. (i) A similar pattern is observed in ovaries mutant for $s p n-D^{2}$, which is required for DSB repair. $(g)$ Mutations in mei-W68 (mei-W6 $8^{l} / \mathrm{mei}^{-}$ $\left.W 68^{k 05603}\right)$, which encodes the Spo11 nuclease that initiates meiotic DSBs, suppress formation of $\gamma$-H2Av foci in region 2 of the germarium. $(h)$ However, mei-W68 does not suppress $\gamma$-H2Av focus formation in armi mutants ( $m e i-W 68^{1} / \mathrm{mei}^{-}$ W68 $8^{\mathrm{k0503}}$; $\mathrm{armi}^{72.1} / \mathrm{armi}^{1}$ ). Projections of six serial $1-\mu \mathrm{m}$ optical sections are shown. Posterior is to the right. Scale bar, $20 \mu \mathrm{m}$. 
A.
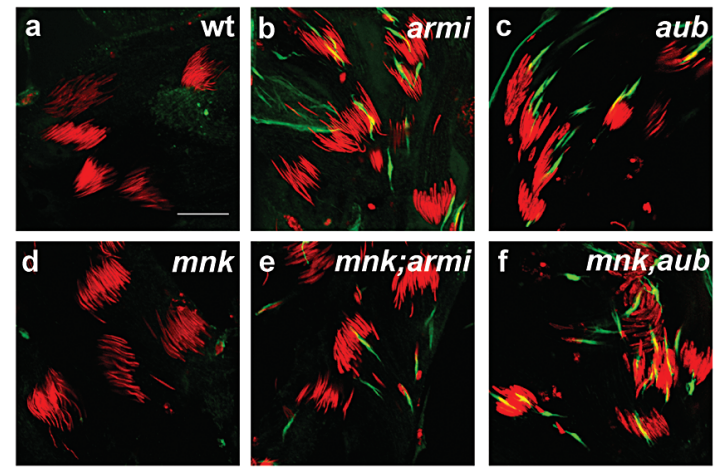

B.
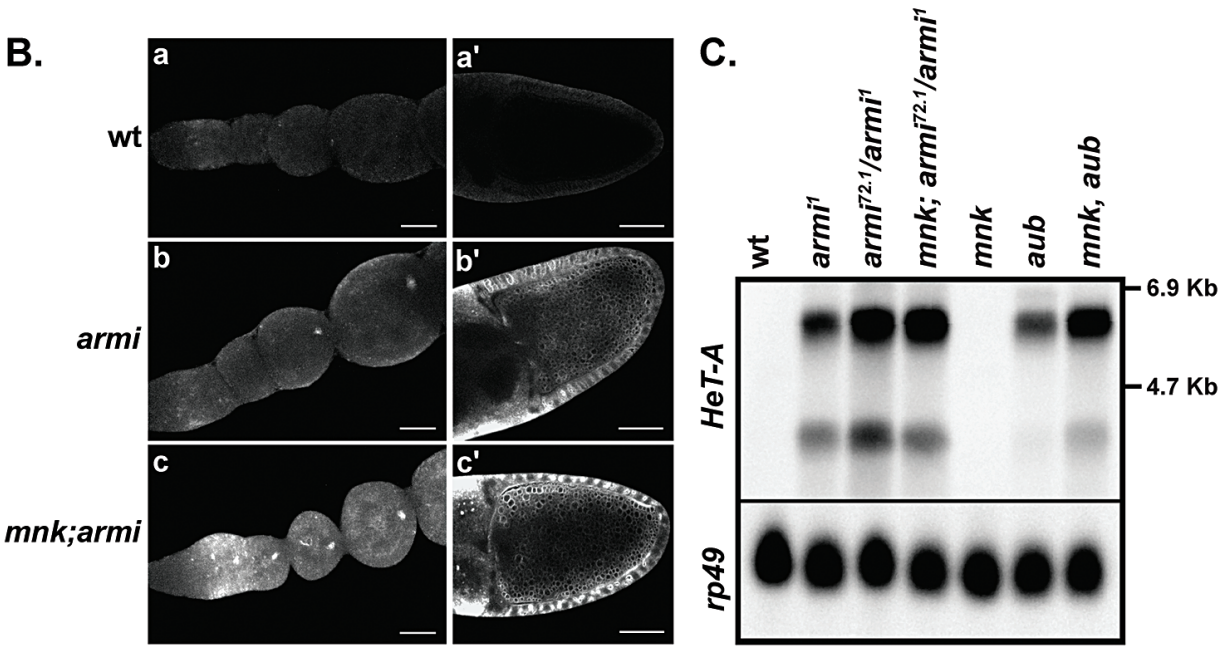

anterior $\longrightarrow$ posterior

Figure 6. The mnk mutation does not suppress defects in rasiRNA function. (A) Silencing of Stellate locus during spermatogenesis. Stellate is not expressed in $(a)$ wild type or $(d) m n k^{p 6}$ mutant testes. However, Stellate is overexpressed, and the protein assembles into

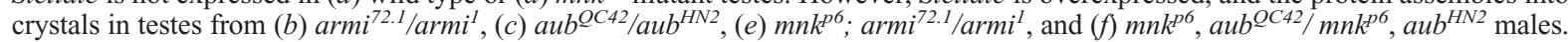
DNA (red) was labeled with TOTO3, and Stellate protein (green) was detected with anti-Stellate antibody. Projections of five serial $1-$ $\mu \mathrm{m}$ optical sections are shown. Scale bar, $20 \mu \mathrm{m}$. (B) FISH analysis of HeT-A retrotransposon expression. $\left(a, a^{\prime}\right)$ In wild-type ovaries, only background levels of HeT-A expression are detected. $\left(b, b^{\prime}\right.$ and $\left.c, c^{\prime}\right)$ In contrast, HeT-A is expressed at high levels in the germline and somatic follicle cells of early and mid-oogenesis stage $a r m i^{72.1} / a r m i^{1}$ and $m n k^{p 6} ; a r m i^{72.1} / a r m i^{1}$ egg chambers. Panels $a, b$, and $c$ are projections of $12-15$ serial $1.5-\mu \mathrm{m}$ optical sections. Panels $a^{\prime}, b^{\prime}$, and $c^{\prime}$ are single optical sections. Posterior is oriented to the right. Scale bars, $20 \mu \mathrm{m}$ for the left panels and $50 \mu \mathrm{m}$ for the right panels. $(C)$ Northern blot for HeT-A. Total ovary RNA samples were resolved on a $1 \%$ agarose-formaldehyde gel, transferred to membrane, and probed for HeT-A transcript. HeT-A transcripts are undetectable in wild-type and $m n k^{p 6}$ samples, but are abundant in RNA derived from armi ${ }^{1}, a_{r m i} i^{2.1} / a^{a r m i} i^{1}, m n k^{p 6} ; a_{r m i}^{72.1} / a r m i^{1}$, $a u b^{Q C 42} / a u b^{H N 2}$ and $m n k^{p 6}$, $a u b^{Q C 42} / a u b^{H N 2}$ mutant ovaries. Ribosomal protein 49 (rp49) was used as a loading control.

telomere formation in Drosophila (Pardue et al. 2005), and $H e T-A$ expression is dramatically de-repressed in armi, $a u b$, and spn-E mutant ovaries (Aravin et al. 2001; Vagin et al. 2004, 2006). HeT-A is not expressed at detectable levels on northern blots of wild-type or $m n k$ RNAs. However, HeT-A transcripts are abundant in armi and $a u b$ mutants (Fig. 6C). Significantly, HeT-A is also overexpressed in $m n k$; $a r m i$ and $m n k$, $a u b$ double mutants (Fig. 6, B and C). In fact, $H e T-A$ expression is higher in the double mutants, relative to the single mutants. FISH analyses indicate that this reflects increased expression in the germ-line and somatic cells of the ovary, during both early and mid-oogenesis (Fig. 6B). Therefore, the $m n k$ mutation does not suppress defects in rasiRNAbased gene silencing during spermatogenesis or oogenesis, leading us to conclude that rasiRNA-based silencing is not required for axis specification.
Taken together, these observations indicate that the critical developmental function for $a r m i$ and $a u b$, and presumably the rasiRNA pathway, is to maintain germ-line genome integrity. The embryonic patterning defects associated with these mutations, in contrast, are due to activation of a ATR/Chk2-dependent DNA damage response that disrupts structural polarization of developing oocyte.

\section{DISCUSSION}

Mutations in the Drosophila armi, spn-E, and $a u b$ genes disrupt oocyte microtubule organization and asymmetric localization of mRNAs and proteins that specify the posterior pole and dorsal-ventral axis of the oocyte and embryo. Mutations in these genes block homology-dependent RNA cleavage and RISC assembly in ovary lysates, RNAi-based gene silencing during 
early embryogenesis, and rasiRNA production and retrotransposon and stellate silencing. These genetic and biochemical studies suggest that siRNAs and/or rasiRNAs are required for embryonic axis specification. However, mutations in the $r 2 d 2, d c r 2$, and ago 2 genes block siRNA function, but do not disrupt the rasiRNA pathway or embryonic axis specification. Defects in the rasiRNA pathway thus appear to trigger the embryonic axis specification defects associated with armi, spn-E, and $a u b$ mutations.

Identifying potential targets for rasiRNA control during axis specification is complex, because the functions for these RNAs are only beginning to emerge. To identify potential target rasiRNAs during axis specification, we focused on previously characterized mutations that also disrupt posterior and dorsal-ventral axis specification. We noted striking similarities between the axis specification defects produced by $a r m i, a u b$, and $s p n-E$ and the patterning defects associated with mutations in meiotic DNA DSB repair pathway, suggesting a mechanistic link. The axis specification defects associated with DNA repair mutants result from activation of an ATR- and Chk2dependent DNA damage signal transduction pathway. We therefore considered two alternative hypotheses: (1) The rasiRNA pathway directly controls axis specification, and ATR/Chk2 activation blocks axis specification by inhibiting this pathway. (2) Mutations that disrupt the rasiRNA pathway activate ATR and Chk2, which disrupt axis specification by targeting rasiRNA-independent processes. We show that the cytoskeletal polarization, RNA localization, and eggshell patterning defects associated with armi and aub are efficiently suppressed by mnk and mei-41, which encode Chk2 and ATR kinase components of the DNA damage signaling pathway. In addition, armi, $a u b$, and spn-E mutants accumulate $\gamma-\mathrm{H} 2 \mathrm{Av}$ foci characteristic of DNA breaks (Fig. 5). Finally, armi mutations trigger Chk2-dependent Vasa phosphorylation (Fig. 4). Significantly, HeT-A and Stellate are overexpressed in $m n k$; $a r m i$ and $m n k, a u b$ double mutants, indicating that axis specification does not directly require rasiRNAbased silencing. Instead, the rasiRNA pathway suppresses DNA damage signaling through ATR and Chk2, which disrupt axis specification by blocking microtubule polarization and Grk signaling from the oocyte to the posterior follicle cells (Fig. 7).

The studies described here also provide new insight into the role of DNA damage signaling in axis specification. As first shown by the Schüpbach lab, meiotic DSB repair mutations trigger Chk2-dependent defects in axis specification and phosphorylation of Vasa (Abdu et al. 2002). Vasa is a putative helicase and translation initiation factor required for axis specification, and Schüpbach and colleagues have proposed that Vasa phosphorylation by Chk2 leads to defects in Grk translation that lead to the observed axial patterning defects (Abdu et al. 2002). Axis specification is initiated early in oogenesis, as the microtubule cytoskeleton reorganizes and Grk signals to the posterior follicle cells. We show that mutations in armi and $a u b$ block initial polarization of the microtubule cytoskeleton and Grk protein localization (Figs. 1 and 3). Furthermore, we find that a null allele of vasa does not

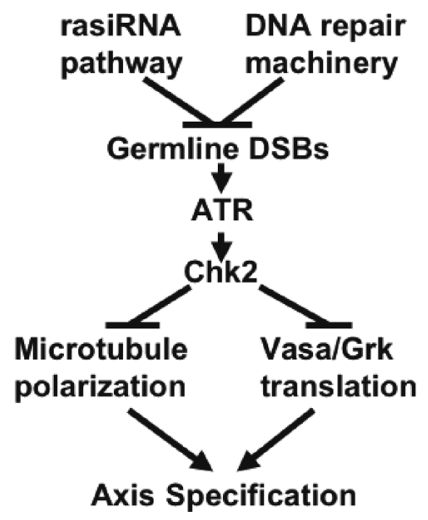

Figure 7. Model for rasiRNA control of axis specification. The rasiRNA pathway and meiotic DSB repair machinery function independently to suppress DSB in the female germ line. Mutations that disrupt either pathway activate a common DNA damage response, mediated by the ATR and Chk2 kinases. Chk2 activation blocks axis specification by disrupting microtubule organization and phosphorylating Vas, an RNA helicase required for axis specification that has been implicated in $g r k$ mRNA translation.

block initial polarization of the microtubule cytoskeleton, indicating that simple loss of Vasa function is not the cause of the initial cytoskeletal polarization defects in armi, $a u b$, and spn-E (C. Klattenhoff and W.E. Theurkauf, unpubl.). Although Vasa is a direct or indirect target of Chk2, these data strongly suggest that Vasa phosphorylation is not the primary cause of the initial axis specification defects, at least in the rasiRNA mutants. Chk2 activation disrupts mitotic microtubule organization and spindle assembly in early embryos (Takada et al. 2003), and the findings reported here indicate that DNA damage disrupts axis specification by activating a Chk2dependent block to microtubule organization, which in turn prevents Grk localization and signaling to the posterior follicle cells.

The role of rasiRNAs in suppressing damage signaling remains to be established. The presence of $\gamma-\mathrm{H} 2 \mathrm{Av}$ foci suggests that DNA DSBs are formed in $a r m i, a u b$, and $s p n-E$ rasiRNA pathway mutations (Fig. 5). In wildtype ovaries, $\gamma-\mathrm{H} 2 \mathrm{Av}$ foci begin to accumulate in region 2 of the germarium, when the Spo11 nuclease (encoded by the mei-W68 gene) initiates meiotic breaks (McKim and Hayashi-Hagihara 1998). The axis specification defects associated with repair mutations are efficiently suppressed by mei-W68 mutations (Ghabrial and Schüpbach 1999; Morris and Lehmann 1999), confirming that meiotic breaks are the source of damage. However, the axis specification defects in armi mutants are not suppressed by mei-W68 (Table 1), and $\gamma-\mathrm{H} 2 \mathrm{Av}$ foci in $a r m i, a u b$, and $s p n-E$ appear to arise somewhat earlier than in wild-type controls. Meiotic DSBs thus do not appear to be the primary source of DNA damage in rasiRNA pathway mutations.

Defects in heterochromatin assembly and a resulting mobilization of retrotransposons could lead to DNA damage and Chk2 activation in rasiRNA pathway mutants (Pal-Bhadra et al. 2004; Vagin et al. 2006). However, silencing of the HeT-A retrotransposon is particularly 
sensitive to rasiRNA pathway mutations, and this element selectively integrates at chromosome ends to form telomeres (Casacuberta and Pardue 2005; Pardue et al. 2005). The rasiRNA mutations could therefore disrupt telomere organization, leading to both loss of silencing and recognition of unprotected chromosome ends by the DSB detection machinery. If this is the case, the $\gamma$-H2Av foci in rasiRNA mutants should colocalize with telomeres. In addition, DNA damage can induce loss of retrotransposon silencing (Bradshaw and McEntee 1989; Scholes et al. 2003; Sacerdot et al. 2005). This raises the possibility that rasiRNAs function in DNA repair, and that mutations in this pathway thus lead to DNA damage that triggers the observed defects in axis specification and loss of retrotransposon silencing. The available data do not distinguish between these alternatives.

In mouse, the piwi-related Argonauts Miwi and Mili bind piRNAs, 30-nucleotide RNAs derived primarily from a single strand that appear to be related to rasiRNAs (Aravin et al. 2006; Girard et al. 2006; Grivna et al. 2006). Mutations in these genes disrupt spermatogenesis and lead to germ-line apoptosis (Kuramochi-Miyagawa et al. 2001; Deng and Lin 2002), which can be induced by DNA damage. We therefore speculate that the primary function for mammalian piRNAs, like Drosophila rasiRNAs, is to suppress DNA damage signaling.

\section{ACKNOWLEDGMENTS}

We thank Kim McKim for $\gamma$-H2Av antibody; Anne Ephrussi for anti-Oskar antibody; and Alla Sigova, Vasia Vagin, and Phil Zamore for HeT-A clones. We also thank Beatrice Benoit and Hanne Varmark for helpful comments on the manuscript; Phil Zamore, Vasia Vagin, Klaus Forstemann, and Yuki Tommari for stimulating discussion and sharing data prior to publication; and Beatrice Benoit for technical support. The Grk monoclonal antibody 1D12, developed by Trudi Schüpbach, was obtained from the Developmental Studies Hybridoma Bank developed under the auspices of the National Institute of Child Health and Human Development and maintained by the University of Iowa, Department of Biological Sciences, Iowa City, Iowa 52242. This work was supported by a grant to W.E.T. from the National Institute of Child Health and Human Development, National Institutes of Health (R01 HD049116).

\section{REFERENCES}

Abdu U., Brodsky M., and Schupbach T. 2002. Activation of a meiotic checkpoint during Drosophila oogenesis regulates the translation of Gurken through Chk2/Mnk. Curr. Biol. 12: 1645 .

Abdu U., Gonzalez-Reyes A., Ghabrial A., and Schupbach T. 2003. The Drosophila spn-D gene encodes a RAD51C-like protein that is required exclusively during meiosis. Genetics 165: 197

Aravin A., Gaidatzis D., Pfeffer S., Lagos-Quintana M., Landgraf P., Iovino N., Morris P., Brownstein M.J., Kuramochi-Miyagawa S., Nakano T., et al. 2006. A novel class of small RNAs bind to MILI protein in mouse testes. Nature 442: 203.
Aravin A.A., Klenov M.S., Vagin V.V., Bantignies F., Cavalli G., and Gvozdev V.A. 2004. Dissection of a natural RNA silencing process in the Drosophila melanogaster germ line. Mol. Cell. Biol. 24: 6742.

Aravin A.A., Naumova N.M., Tulin A.V., Vagin V.V., Rozovsky Y.M., and Gvozdev V.A. 2001. Double-stranded RNA-mediated silencing of genomic tandem repeats and transposable elements in the D. melanogaster germline. Curr. Biol. 11: 1017.

Bartek J. and Lukas J. 2003. Chk1 and Chk2 kinases in checkpoint control and cancer. Cancer Cell 3: 421.

Bartek J., Falck J., and Lukas J. 2001. CHK2 kinase - A busy messenger. Nat. Rev. Mol. Cell Biol. 2: 877.

Bernstein E., Kim S.Y., Carmell M.A., Murchison E.P., Alcorn H., Li M.Z., Mills A.A., Elledge S.J., Anderson K.V., and Hannon G.J. 2003. Dicer is essential for mouse development. Nat. Genet. 35: 215.

Bradshaw V.A. and McEntee K. 1989. DNA damage activates transcription and transposition of yeast Ty retrotransposons. Mol. Gen. Genet. 218: 465.

Casacuberta E. and Pardue M.L. 2005. HeT-A and TART, two Drosophila retrotransposons with a bona fide role in chromosome structure for more than 60 million years. Cytogenet. Genome Res. 110: 152.

Cook H., Koppetsch B., Wu J., and Theurkauf W. 2004. The Drosophila SDE3 homolog armitage is required for oskar mRNA silencing and embryonic axis specification. Cell 116: 817.

Deng W. and Lin H. 2002. miwi, a murine homolog of piwi, encodes a cytoplasmic protein essential for spermatogenesis. Dev. Cell 2: 819 .

Deshpande G., Calhoun G., and Schedl P. 2005. Drosophila argonaute- 2 is required early in embryogenesis for the assembly of centric/centromeric heterochromatin, nuclear division, nuclear migration, and germ-cell formation. Genes Dev. 19: 1680.

Forstemann K., Tomari Y., Du T., Vagin V.V., Denli A.M., Bratu D.P., Klattenhoff C., Theurkauf W.E., and Zamore P.D. 2005. Normal microRNA maturation and germ-line stem cell maintenance requires Loquacious, a double-stranded RNAbinding domain protein. PLoS Biol. 3: e236.

Fukagawa T., Nogami M., Yoshikawa M., Ikeno M., Okazaki T., Takami Y., Nakayama T., and Oshimura M. 2004. Dicer is essential for formation of the heterochromatin structure in vertebrate cells. Nat. Cell Biol. 6: 784.

Galiana-Arnoux D., Dostert C., Schneemann A., Hoffmann J.A., and Imler J.L. 2006. Essential function in vivo for Dicer-2 in host defense against RNA viruses in Drosophila. Nat. Immunol. 7: 590 .

Ghabrial A. and Schupbach T. 1999. Activation of a meiotic checkpoint regulates translation of Gurken during Drosophila oogenesis. Nat. Cell Biol. 1: 354.

Ghabrial A., Ray R.P., and Schupbach T. 1998. okra and spindle- $B$ encode components of the RAD52 DNA repair pathway and affect meiosis and patterning in Drosophila oogenesis. Genes Dev. 12: 2711.

Giraldez A.J., Cinalli R.M., Glasner M.E., Enright A.J., Thomson J.M., Baskerville S., Hammond S.M., Bartel D.P., and Schier A.F. 2005. MicroRNAs regulate brain morphogenesis in zebrafish. Science 308: 833.

Girard A., Sachidanandam R., Hannon G.J., and Carmell M.A. 2006. A germline-specific class of small RNAs binds mammalian Piwi proteins. Nature 442: 199.

Gong W.J., McKim K.S., and Hawley R.S. 2005. All paired up with no place to go: Pairing, synapsis, and DSB formation in a balancer heterozygote. PLoS Genet. 1: e67.

Gonzalez-Reyes A., Elliott H., and St. Johnston D. 1995. Polarization of both major body axes in Drosophila by gurken-torpedo signalling. Nature 375: 654.

Grishok A., Pasquinelli A.E., Conte D., Li N., Parrish S., Ha I., Baillie D.L., Fire A., Ruvkun G., and Mello C.C. 2001. Genes and mechanisms related to RNA interference regulate expression of the small temporal RNAs that control C. elegans developmental timing. Cell 106: 23. 
Grivna S.T., Beyret E., Wang Z., and Lin H. 2006. A novel class of small RNAs in mouse spermatogenic cells. Genes Dev. 20: 1709.

Gvozdev V.A., Aravin A.A., Abramov Y.A., Klenov M.S., Kogan G.L., Lavrov S.A., Naumova N.M., Olenkina O.M., Tulin A.V., and Vagin V.V. 2003. Stellate repeats: Targets of silencing and modules causing cis-inactivation and trans-activation. Genetica 117: 239.

Hannon G.J. 2002. RNA interference. Nature 418: 244.

Hatfield S.D., Shcherbata H.R., Fischer K.A., Nakahara K., Carthew R.W., and Ruohola-Baker H. 2005. Stem cell division is regulated by the microRNA pathway. Nature 435: 974.

Hirao A., Cheung A., Duncan G., Girard P.M., Elia A.J., Wakeham A., Okada H., Sarkissian T., Wong J.A., Sakai T., et al. 2002. Chk2 is a tumor suppressor that regulates apoptosis in both an ataxia telangiectasia mutated (ATM)-dependent and an ATM-independent manner. Mol. Cell. Biol. 22: 6521.

Hutvagner G. and Zamore P.D. 2002. A microRNA in a multiple-turnover RNAi enzyme complex. Science 297: 2056.

Jang J.K., Sherizen D.E., Bhagat R., Manheim E.A., and McKim K.S. 2003. Relationship of DNA double-strand breaks to synapsis in Drosophila. J. Cell Sci. 116: 3069.

Kuramochi-Miyagawa S., Kimura T., Yomogida K., Kuroiwa A., Tadokoro Y., Fujita Y., Sato M., Matsuda Y., and Nakano T. 2001. Two mouse piwi-related genes: miwi and mili. Mech. Dev. 108: 121.

Lee Y.S., Nakahara K., Pham J.W., Kim K., He Z., Sontheimer E.J., and Carthew R.W. 2004. Distinct roles for Drosophila Dicer-1 and Dicer-2 in the siRNA/miRNA silencing pathways. Cell 117: 69.

Madigan J.P., Chotkowski H.L., and Glaser R.L. 2002. DNA double-strand break-induced phosphorylation of Drosophila histone variant $\mathrm{H} 2 \mathrm{Av}$ helps prevent radiation-induced apoptosis. Nucleic Acids Res. 30: 3698.

McKim K.S. and Hayashi-Hagihara A. 1998. mei-W68 in Drosophila melanogaster encodes a Spo11 homolog: Evidence that the mechanism for initiating meiotic recombination is conserved. Genes Dev. 12: 2932.

Modesti M. and Kanaar R. 2001. DNA repair: Spot(light)s on chromatin. Curr. Biol. 11: R229.

Morris J. and Lehmann R. 1999. Drosophila oogenesis: Versatile spn doctors. Curr. Biol. 9: R55.

Neuman-Silberberg F.S. and Schupbach T. 1993. The Drosophila dorsoventral patterning gene gurken produces a dorsally localized RNA and encodes a TGF alpha-like protein. Cell 75: 165.

Oikemus S.R., McGinnis N., Queiroz-Machado J., Tukachinsky H., Takada S., Sunkel C.E., and Brodsky M.H. 2004. Drosophila atm/telomere fusion is required for telomeric localization of HP1 and telomere position effect. Genes Dev. 18: 1850 .

Okamura K., Ishizuka A., Siomi H., and Siomi M.C. 2004. Distinct roles for Argonaute proteins in small RNA-directed RNA cleavage pathways. Genes Dev. 18: 1655 .

Pal-Bhadra M., Leibovitch B.A., Gandhi S.G., Rao M., Bhadra U., Birchler J.A., and Elgin S.C. 2004. Heterochromatic silencing and HP1 localization in Drosophila are dependent on the RNAi machinery. Science 303: 669.

Pardue M.L., Rashkova S., Casacuberta E., DeBaryshe P.G., George J.A., and Traverse K.L. 2005. Two retrotransposons maintain telomeres in Drosophila. Chromosome Res. 13: 443.

Provost P., Silverstein R.A., Dishart D., Walfridsson J., Djupedal I., Kniola B., Wright A., Samuelsson B., Radmark O., and Ekwall K. 2002. Dicer is required for chromosome segregation and gene silencing in fission yeast cells. Proc. Natl. Acad. Sci. 99: 16648.
Redon C., Pilch D., Rogakou E., Sedelnikova O., Newrock K., and Bonner W. 2002. Histone $\mathrm{H} 2 \mathrm{~A}$ variants $\mathrm{H} 2 \mathrm{AX}$ and H2AZ. Curr. Opin. Genet. Dev. 12: 162.

Riechmann V. and Ephrussi A. 2001. Axis formation during Drosophila oogenesis. Curr. Opin. Genet. Dev. 11: 374.

Rogakou E.P., Pilch D.R., Orr A.H., Ivanova V.S., and Bonner W.M. 1998. DNA double-stranded breaks induce histone H2AX phosphorylation on serine 139. J. Biol. Chem. 273: 5858.

Roth S., Neuman-Silberberg F.S., Barcelo G., and Schupbach T. 1995. cornichon and the EGF receptor signaling process are necessary for both anterior-posterior and dorsal-ventral pattern formation in Drosophila. Cell 81: 967.

Sacerdot C., Mercier G., Todeschini A.L., Dutreix M., Springer M., and Lesage P. 2005. Impact of ionizing radiation on the life cycle of Saccharomyces cerevisiae Ty1 retrotransposon. Yeast 22: 441

Scholes D.T., Kenny A.E., Gamache E.R., Mou Z., and Curcio M.J. 2003. Activation of a LTR-retrotransposon by telomere erosion. Proc. Natl. Acad. Sci. 100: 15736.

Schupbach T. 1987. Germ line and soma cooperate during oogenesis to establish the dorsoventral pattern of egg shell and embryo in Drosophila melanogaster. Cell 49: 699.

Spradling A.C. 1993. Germline cysts: Communes that work. Cell 72: 649.

Stapleton W., Das S., and McKee B.D. 2001. A role of the Drosophila homeless gene in repression of Stellate in male meiosis. Chromosoma 110: 228.

Styhler S., Nakamura A., Swan A., Suter B., and Lasko P. 1998. vasa is required for GURKEN accumulation in the oocyte, and is involved in oocyte differentiation and germline cyst development. Development 125: 1569.

Takada S., Kelkar A., and Theurkauf W.E. 2003. Drosophila checkpoint kinase 2 couples centrosome function and spindle assembly to genomic integrity. Cell 113: 87 .

Theurkauf W.E., Alberts B.M., Jan Y.N., and Jongens T.A. 1993. A central role for microtubules in the differentiation of Drosophila oocytes. Development 118: 1169.

Theurkauf W.E., Smiley S., Wong M.L., and Alberts B.M. 1992. Reorganization of the cytoskeleton during Drosophila oogenesis: Implications for axis specification and intercellular transport. Development 115: 923.

Tomari Y., Matranga C., Haley B., Martinez N., and Zamore P.D. 2004a. A protein sensor for siRNA asymmetry. Science 306: 1377.

Tomari Y., Du T., Haley B., Schwarz D., Bennett R., Cook H., Koppetsch B., Theurkauf W., and Zamore P.D. 2004b. RISC assembly defects in the Drosophila RNAi mutant armitage. Cell 116: 831 .

Vagin V.V., Klenov M.S., Kalmykova A., Stolyarenko A.D., Kotelnikov R.N., and Gvozdev V. 2004. The RNA interference proteins and vasa locus are involved in the silencing of retrotransposons in the female germline of Drososophla melanogaster. RNA Biol. 1: 54.

Vagin V.V., Sigova A., Li C., Seitz H., Gvozdev V., and Zamore P.D. 2006. A distinct small RNA pathway silences selfish genetic elements in the germline. Science 313: 320.

Volpe T., Schramke V., Hamilton G.L., White S.A., Teng G., Martienssen R.A., and Allshire R.C. 2003. RNA interference is required for normal centromere function in fission yeast. Chromosome Res. 11: 137.

Wang X.H., Aliyari R., Li W.X., Li H.W., Kim K., Carthew R., Atkinson P., and Ding S.W. 2006. RNA interference directs innate immunity against viruses in adult Drosophila. Science 312: 452.

Wassenegger M. 2005. The role of the RNAi machinery in heterochromatin formation. Cell 122: 13. 


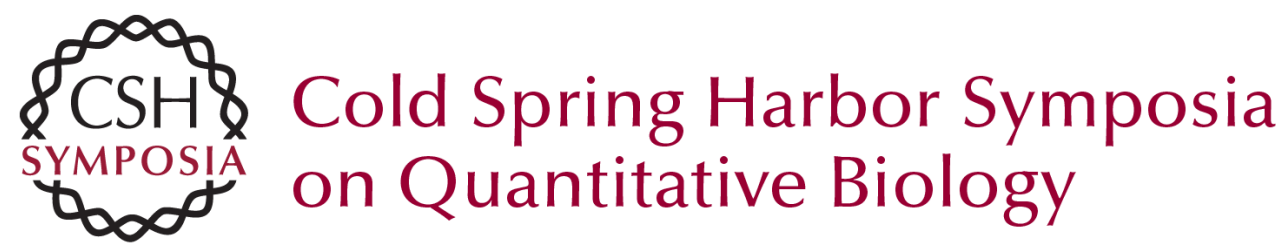

\section{rasiRNAs, DNA Damage, and Embryonic Axis Specification}

W.E. THEURKAUF, C. KLATTENHOFF, D.P. BRATU, et al.

Cold Spring Harb Symp Quant Biol 2006 71: 171-180

Access the most recent version at doi:10.1101/sqb.2006.71.066

References This article cites 62 articles, 22 of which can be accessed free at: http://symposium.cshlp.org/content/71/171.full.html\#ref-list-1

\section{License}

Email Alerting Receive free email alerts when new articles cite this article - sign up in Service the box at the top right corner of the article or click here. 